\title{
Consolidación del pensamiento pedagógico en tomo al deporte
}

\section{Clara Lourdes Peña Castro Rafael Antonio Morales}

\section{Resumen}

El reconocimiento de los actores y agencias del conocimiento, la construcción del pensamiento por parte de los gestores y las diversas formas con las que hoy en día se organizan los saberes producidos, contribuyen a que se admita la sistematización como modalidad investigativa en el ámbito de las ciencias sociales, para interpretar y comprender las prácticas que se evidencian en los hechos y procesos socio culturales, permitiendo entender la realidad y cotidianidad para la proyección de posibles acciones formativas. El asunto determinante para el desarrollo de procesos educativos y formativos desde la academia, es visualizar las formas de actualizarse frente a la realidad y su cotidianidad donde socialmente se trasluce toda una serie de expresiones; a este proceso se hace referencia en el presente artículo, avance parcial de la investigación que actualmente se encuentra en el tercer momento de su desarrollo y se denomina: «Discursos, saberes y prácticas del deporte contemporáneo en Bogotá y Cundinamarca. Una experiencia de sistematización». ${ }^{1}$

Palabras clave: discurso, saber, práctica, deporte, cultura, pedagogía, tecnología, sistematización, módulos temáticos, interpretación, comprensión, explicación, diálogo cultural, diálogo de saberes, experiencia, resignificación.

\section{Alusión al proyecto investigativo}

En la actualidad el deporte se detecta como algo evidente ligado a un modo de existencia de los seres humanos, que inevitablemente participan desde la diversidad de roles, creencias, imaginarios e ideologías, lo que dificulta el estudio del fenómeno deportivo, ya que la reflexión educativa y formativa se amplia hacia horizontes culturales abiertos que no siempre están circunscritos única y exclusivamente en los espacios escolarizados.

Discursos, saberes y prácticas circulan en el contexto deportivo como si estuviesen inscritos «espontáneamente» en los desarrollos sociales y culturales, de tal forma que pareciera que éste dispositivo de la modernidad amplificara su existencia hacia delante y atrás, afectando el desarrollo humano y deportivo, y por supuesto, la evolución histórica de la educación física pasada y por venir.

¿Cómo consolidar el pensamiento pedagógico que dinamice el sentido de horizonte en torno al deporte y' en qué forma sistematizar el saber construido que resulta de la experiencia, con el propósito de identificar, reconocer y difundir la producción elaborada por fuera de la academia? Entre otros objetivos, a nivel general son algunos de los propósitos del proyecto investigativo «Discursos saberes y prácticas del deporte contemporáneo en Bogotá y Cundinamarca. Una experiencia de sistematización»,

\footnotetext{
* Profesores de la Facultad de Educación Física, Universidad Pedagógica Nacional. Investigadores del proyecto avalado por el CIUP con el código FEF 015-01

${ }^{1}$ Investigación avalada por el CIUP
} 
adelantado en la Facultad de Educación Física, con recursos asignados por la División de Gestión de Proyectos —CIUP_ de la Universidad Pedagógica Nacional.

Las relaciones que se establecen entre el estudio de la realidad producida en las prácticas profesionales, el interés por develar su sentido, y la reflexión-explicación académica, hacen pensar que las teorías investigativas más adecuadas para el desarrollo del proyecto y con las cuales se puede producir un conocimiento acerca de la realidad estudiada, son las de corte social y la utilización de los enfoques histórico-hermenéutico y crítico-social (Carr y Kemmis, 1986).

Entonces, en la preocupación por identificar, reflexionar y estudiar la experiencia de los profesionales que se desempeñan en el contexto deportivo, se hace necesario seleccionar la metodología que más se ajuste a las dinámicas sociales y culturales en las cuales se desarrollan las motivaciones relacionadas con el deporte y los actores que en él intervienen.

Pero a la preocupación anterior, simultáneamente se suma la inquietud por alentar la discusión académica y continuada sobre el objeto de estudio y sus diferentes problemáticas, concibiéndose para ello la idea de constituir y consolidar un grupo que dinamice procesos investigativos; por tanto, metodológicamente se recurre a los paradigmas que posibiliten, por un lado, la interpretación, comprensión y sistematización de los discursos, saberes y prácticas, y de igual forma, la conformación y ampliación del equipo investigador ${ }^{2}$.

Teniendo en cuenta lo anterior, el proyecto investigativo se impone una doble función: en primer lugar, sensibilizar a la comunidad sobre la importancia y pertinencia de reconocer el saber-conocimiento construido por los actores sociales del deporte como resultado de la reflexión sobre la experiencia (Schön, 1983), y en segundo término, responder a la necesidad de conformar comunidades académicas que se interesen por los aspectos educativos y formativos, con miras a cualificar los procesos de formación profesional en el campo específico de la educación física en general y el deporte en particular.

\section{Sobre el proceso metodológico investigativo}

Desde la evolución de los procesos metodológicos de la investigación, se establecen cuatro momentos para su desarrollo, así:

1. El primer momento hace referencia a la organización y diseño de los instrumentos, teniendo en cuenta inicialmente ${ }^{3}$ las dimensiones de análisis que a continuación se relacionarán: a) Concepto de deporte desde la dimensión pedagógica (educativa y formativa); b) Concepto de deporte desde la dimensión cultural; c) Concepto de deporte desde la dimensión tecnológica, todas con miras a propiciar el diálogo cultural con los actores del deporte.

\footnotetext{
${ }^{2}$ En relación con este aspecto, en el mes de enero pasado se vincularon a la investigación los profesores de planta de la Facultad de Educación física, Nelly teresa Martínez y Efraín Serna caldas

${ }^{3}$ El término «inicialmente» hace referencia a la posibilidad de ampliar las categorías o dimensiones de estudio, debido a las características de los actores entrevistados, a las dinámicas, imaginarios y pensamientos que tienen los actores sobre lo que significa deporte o a los mismos procesos que generan durante el desarrollo del trabajo investigativo
} 
2. El segundo momento está relacionado con el contacto inicial para entablar el diálogo de saberes por medio de entrevistas semiestructuradas ${ }^{4}$, debidamente registradas, posteriormente transcritas y clasificadas en siete Módulos Temáticos ${ }^{5}$ que recogen lo expresado por 53 actores entrevistados.

3. El tercer momento hace alusión a la organización de talleres que se han denominado de confrontación; el propósito principal de los Talleres de Confrontación es posibilitar el diálogo cultural por medio del encuentro, reconocimiento e interpretación entre los actores y sus textos respectivos, y simultáneamente como producto de esos diálogos, conformar el grupo investigador ${ }^{6}$ que organice el Congreso Regional de Actores Sociales del Deporte contemporáneo.

4. El cuarto momento se relaciona en primera instancia, con la sistematización, entendida ésta como la modalidad de producción de conocimiento con carácter participativo, que busca la cualificación, transformación y potenciación a partir de la reconstrucción de una práctica social y su intervención crítica para aportar al campo de conocimiento en que se ubica dicha práctica (Torres, 2002). En segundo término, el cuarto momento de la investigación se relaciona con la socialización de los resultados del Proyecto investigativo.

\section{Importancia de los Módulos Temáticos en el contexto de la investigación}

Al hacer referencia al sentido sobre lo que representa el deporte desde las dimensiones de estudio propuestas por el Proyecto investigativo, es evidente que se debe partir del relato y la historia de vida de aquellas personas que se han interesado por el hecho social deportivo.

La decisión de diseñar e implementar los Módulos Temáticos, obedece a la estrategia didáctica que posibilita y facilita el pretexto para el encuentro entre actores, quienes a partir de la lectura, interpretación, comprensión y explicación de las representaciones allí consignadas, tienen la oportunidad de conocer la memoria individual, o acto del recuerdo en el ámbito de lo privado y a su vez, la memoria colectiva que se asume como interpretación de hechos que se ubican en el campo de lo público, siendo ampliamente compartidos por el grupo social, pero en la mayoría de los casos y en gran parte, desconocida y sin sistematizar por la academia.

Con la lectura de los Módulos Temáticos se pretende entender las relaciones entre la historia del actor social del deporte como sujeto de saber, y los procesos de organización y desarrollo de las prácticas deportivas en su localidad o región; es decir, se propone reconstruir el sentido y significado del deporte desde el testimonio efectuado por los actores, y por tanto, tomar conciencia de dicho acto como el evento que integra al actor a los procesos sociales y que a su vez, lo transforma y evoluciona de acuerdo con los intereses y expectativas tanto personales, como de los grupos sociales a los cuales pertenece.

\footnotetext{
${ }^{4}$ En cuyo desarrollo, a través de las narraciones de los actores, surgen espontáneamente especies de subdimensiones con relación a: a) relatos e historias de vida: intereses y expectativas del deporte a nivel personal; b) Concepto y perspectivas de la práctica deportiva; c) concepto de cultura organizacional en el deporte: problemas y expectativas; d) sugerencias y aportes alas facultades de educación física, recreación y deporte, responsables de la formación profesional ${ }^{5}$ Un módulo temático, por cada una de las dimensiones mencionadas

${ }^{6}$ Uno de los resultados del primer Taller de Confrontación adelantado el pasado 24 de agosto, es la vinculación al proyecto investigativo de la profesional Ximena Herrera, Profesora de la facultad de Educación Física de La Universidad Pedagógica Nacional.
} 
En el marco de la investigación los Módulos Temáticos se constituyen en la base que apoya la interpretación, posibilitan el encuentro para el diálogo, facilitan la constitución del grupo investigador, propician las dinámicas metodológicas a sugerir por parte de los lectores intérpretes para el estudio del material recolectado y viabilizan la organización de los Talleres y Congreso Regional. Desde lo anterior, a continuación se presenta una breve explicación sobre cada uno de los Módulos Temáticos:

Módulo 1: Relatos e historias de vida: intereses y expectativas del deporte a nivel personal.

En la acción investigativa de las ciencias sociales, el uso de los relatos $y$ las historias de vida se ha constituido en una herramienta de observación y comprensión de los niveles de evolución y estructuración de las personas en los distintos ámbitos de la vida cotidiana y social.

Desde una orientación pragmática, el relato y la historia de vida se conciben como un medio para entender la manera como las personas piensan la vida $y$ sobre las posibilidades reales para transformar su medio; por tanto, el relato $y$ la historia de vida son consideradas como una técnica para construir teoría sobre la comprensión de lo que es la eistencia en el individuo.

En consecuencia, «relatos e historias de vida: intereses y expectativas personales con relación al deporte», como su titulo lo indica, es un relato de vida, una historia y en ocasiones una biografía que caracteriza al actor social del deporte desde sus sueños, utopías, realizaciones, logros y aspiraciones en cuanto a las prácticas deportivas y su correspondiente sentido.

\section{Módulo 2: concepto de deporte desde la dimensión cultural}

El concepto de deporte contemporáneo se entiende como un modelo cultural con estructura social que engloba valores, normas, sanciones, conocimientos posiciones sociales con la marcada tendencia de socializar al individuo en la práctica deportiva. Quiere decir que el acto deportivo, incluye principios, valores, políticas, ideologías, aspectos éticos, estéticos y dimensiones que proyectan al ser humano en su integralidad y sus características culturales. (Elías y Dunning, 1995).

El sentido y significado del deporte desde la dimensión cultural, representa el interés académico de la investigación por reflexionar y resaltar la presencia, importancia y fuerza del imaginario y la realidad deportiva en nuestra sociedad, particularmente en Bogotá y Cundinamarca; busca evidenciar los imaginarios de la realidad del deporte, con los cuales tanto los actores sociales como las instituciones $y$ agencias del deporte, representan el sentido y significado cultural del acto deportivo.

\footnotetext{
Una primera aproximación al concepto de cultura, parte de la necesidad de tomar conciencia del carácter polisémico y la heterogeneidad de las acepciones; esta situación motiva a reconocer que el significado exacto de este término, es tema de controversia para los diferentes profesionales que se dedican a su interpretación.
}

Es importante tener en cuenta que el deporte es ante todo un producto cultural como tal debe entenderse (Blanchard y Cheska, 1986), lo que significa, que en sí mismo, el 
deporte es cultura; por otra parte, se asume el deporte como una parte integral de la sociedad y su cultura, que afecta de formas muy variadas y en ocasiones importantes, la vida de los individuos y de los grupos sociales.

En síntesis, el Módulo Temático 2 se convierte en el medio para interpretar, comprender y explicar el conjunto de creencias, mitos, saberes y prácticas del deporte existentes en el medio, colaborando en el acercamiento hacia las maneras de pensar, actuar y sentir el deporte como una opción cultural que caracterizan determinada localidad o región.

\section{Módulo 3: Concepto de deporte desde la dimensión pedagógica}

Producto del paradigma interpretativo, el horizonte de sentido del deporte se fundamenta en la comprensión y apropiación de lo que significa la acción deportiva como unidad de análisis, pero no sólo como una «abstracción teórica», sino también como principio explicativo de la dimensión pedagógica de la práctica deportiva actual.

El concepto de pedagogía que tradicionalmente se ha tenido en cuenta para dialogar con el saber recreado por los actores sociales del deporte, es aquél concepto que la asume como disciplina que conceptualiza, aplica y experiencia los conocimientos referentes a la enseñanza de los saberes específicos en las diferentes culturas; teniendo en cuenta que la pedagogía no es sólo un discurso acerca de la enseñanza, sino también una práctica cuyo campo de aplicación es el discurso (Zuluaga, 1999). El proyecto investigativo tiene en cuenta las tendencias, enfoques, posturas e identidades pedagógicas y educativas existentes en el contexto local. Por consiguiente, la interpretación de la dimensión pedagógica integra tanto los saberes elaborados por la experiencia, como el nivel de reflexión y análisis de la pedagogía, la educación, la enseñanza y la formación.

En este contexto, el diálogo en torno a la dimensión pedagógica y educativa del deporte, debe tener en cuenta los propósitos y fines que motivan las prácticas deportivas en las comunidades, a partir de unos intereses $\sim$ expectativas que se relacionan con el concepto de hombre y sociedad.

\section{Módulo 4: concepto de deporte desde la dimensión tecnológica}

La técnica como tal, ha acompañado siempre la vida del ser humano; así, un determinado grado de desarrollo de la técnica, es por tanto indispensable para la supervivencia y bienestar de cualquier grupo humano. Una primera aproximación conceptual tiene que ver con los niveles de creatividad e innovación para orientar con eficacia una actividad cualquiera, lo que conlleva, en ocasiones, a niveles de apropiación de procesos alterativos de la acción.

Sin embargo, la creatividad e innovación en el contexto de la producción técnica, no hace referencia a cualquier cosa o habilidad con que pueda hacerse, más bien, alude a la característica que está integrada a un conjunto de reglas o procedimientos aptos para conseguir unos productos que responden a unos propósitos predeterminados. Desde el punto de vista racional, la técnica es relativamente ignorada intelectualmente; sin embargo, debe recordarse que las técnicas son explicadas y comunicadas por diferentes medios y formas, lo que no excluye su forma particular de generar un saber sobre los 
procedimientos que cada cultura construye, con el propósito de resolver problemas relacionados con su cotidianidad.

Independientemente de las creencias particulares y/o populares, el estudio de la técnica puede conducir su modificación o corrección por medio de la reflexión sobre la acción misma; es decir, lo que ante todo se necesita con relación al estudio de la técnica, es crear el sistema de conceptos dentro de los cuales puedan plantearse los problemas básicos de la tecnología.

Así, la tecnología como el estudio de los procedimientos técnicos de una determinada especificidad que conduce a la producción propia de sus características, determina la existencia de un pensamiento que ha sido creado y recreado a través de la experiencia por los actores, quienes demuestran que los saberes construidos contribuyen en cierta medida al desarrollo x evolución de las diferentes prácticas.

\section{Módulo 5: Concepto de práctica deportiva}

Se busca con el desarrollo conceptual sobre las prácticas deportivas, establecer una relación directa con lo trascendental del acto deportivo, a partir del pensamiento que gira en torno a la acción con sentido y razón, que pretende dar forma a éstas, y a la vez, al espacio de construcción del criterio y el pensamiento que busca la solución a problemas sociales, educativos y culturales, tanto de las prácticas deportivas, como de un grupo social en particular.

Se asume el proceso de construcción conceptual de las prácticas deportivas desde el sentido pragmático que integra la acción, el pensamiento, las ideas, las razones y el sentido común; es decir, el proceso de lectura e interpretación de lo que dice y expresa el actor social con relación a dichas prácticas; tiene como propósito observar la actitud de adaptación a principios, necesidades, resultados, consecuencias y hechos que genera el acto deportivo.

En otras palabras, se trata de poner en escena los aspectos prácticos antes que crear teorías; observar la forma como el actor social del deporte asume el sentido de la práctica deportiva, colabora para evidenciar sus consecuencias, además de explicar las diferencias tanto de las acciones deportivas, como de los efectos que éstas producen.

Las prácticas como espacio dialéctico, posibilitan la creación de sueños, utopías e ideales que enriquecen los procesos tendientes a la liberación y la solución de los problemas de la sociedad y el hombre, resignificando la reflexión acerca de lo que se hace $y$ se construye en el campo profesional y del conocimiento, $y$ la toma de conciencia de la realidad del individuo los grupos sociales que practican deporte.

\section{Módulo 6: Concepto de cultura organizacional en el deporte}

El concepto de cultura organizacional del deporte es en si mismo una iniciativa de carácter académico que pretende comprender las construcciones, imaginarios $y$ representaciones relacionadas directamente con el planeamiento, gestión y desarrollo como consecuencia de la configuración del modo de sentir, pensar y vivir el acto deportivo en el marco de la organización institucional-empresarial. 
En general, se concibe el sistema deportivo como un movimiento social autónomo que posee la capacidad de crear su propio sistema de funcionamiento (Jeu, 1988), a partir de una integración e interpretación del mundo, la vida y las cosas externas al hombre, que incluyen actitudes, motivaciones, convicciones y valores de los grupos sociales, dando como resultado una forma muy particular en la organización de la gestión y administración del deporte.

Desde el anterior sentido, las teorías administrativas contemporáneas conceptúan los procesos humanos empresariales a partir de la idea de competitividad $y$ calidad, con miras a establecer estrategias de desarrollo, evolución y sobrevivencia empresarial. Es necesario, entonces, reconocer y valorar el conocimiento-saber producido por la experiencia, con relación a la cultura organizacional por parte de los actores sociales del deporte, como consecuencia de la toma de conciencia sobre la relevancia e importancia de su sistema organizativo y político, para el proceso de evolución y transformación de los grupos humanos y la sociedad.

\section{Módulo 7: Sugerencias y aportes a las facultades de educación física, recreación y deporte, responsables de la formación profesional}

El módulo recoge las inquietudes que surgen como el producto de la experiencia acumulada por los actores en los diferentes eventos que tienen que ver con el fenómeno deportivo. Por medio de las expresiones y manifestaciones, los actores que intervienen en la investigación hacen un llamado a las instituciones educativas de nivel superior, con relación a las acciones formativas por adelantar en la academia con los futuros estudiantes de la educación física y el deporte; de esta forma se tiene en cuenta que los posteriores profesionales son quienes están en posibilidad de gestar cambios relacionados con las dimensiones de estudio sobre los cuales fueron entrevistados.

Desde lo anterior, puede decirse que el deporte es un fenómeno social que sugestiona diversos comportamientos y actitudes en los seres humanos; por tanto, la academia desde sus posibilidades teóricas y prácticas relacionadas con la docencia, el entrenamiento, la educación y la formación de los futuros profesionales, debe visualizar, fundamentar y orientar el horizonte de sentido que se le asignará al deporte, para que dichas prácticas contribuyan a que el desarrollo humano sea cada vez más humano.

\section{Referencias bibliográficas}

BARREAU, J y MORNE, J. (1998). Epistemología y antropología del deporte, Barcelona, Ed. Doyma.

BLANCHARD, K y CHESKA, A. (1986). Antropología del deporte, Barcelona, Ed. Bellaterra.

CAGIGAL, J. M. (1968). Cultura intelectual; cultura física, Buenos Aires, Ed. Kapelusz.

CARR, W y KEMMIS, S. (1986). Teoría crítica de la enseñanza. La investigaciónacción en la formación del profesorado, Barcelona, Ed. Morata. 
CIFUENTES, R. M. (1999). La sistematización de la práctica del trabajo social, Argentina, Ed Lumen / humanitas.

CUEVAS, P (2001). «El relato y las historias de vida en el campo de las representaciones». En: Revista Aportes, No. 56, Bogotá, Ed. Dimensión educativa.

ELIAS, N. y DUNNING, E. (1995). Deporte y ocio en el proceso de la civilización, México, Fondo de Cultura Económica.

GARCIA CANCLINI, N. (1990). Culturas híbridas. Estrategias para entrar y salir de la modernidad, México, Ed. Grijalbo.

GODAR, F. \& CABANÉS, R. Traducción: SILVA, M. (1995). Uso de las historias de vida en las ciencias sociales». En: cuadernos del Centro de Investigaciones sobre dinámica social Bogotá, Universidad Externado de Colombia.

JEU, B. (1988). Análisis del deporte, Barcelona, Ed. Bellaterra.

HARRIS, M. (1990). Antropología Cultural Madrid, Alianza Editorial S.A.

McClAREN, P. (1994). Pedagogía critica, resistencia cultural; producción del deseo, Buenos Aires, Ed. Aique.

MANDELL, R. D. (1984). Historia cultural del deporte, Barcelona, Ed. Bellaterra.

MERINO, J. (1997). Programas de animación sociocultural: tres instrumentos para su diseño y evaluación. Madrid. Ed. Narcea.

NOVAK, J. y GOWIN, D. B. (1988). Aprendiendo a aprender Barcelona, Ed. Martínez Roca, S.A.

RODRÍGUEZ LOPEZ, J. (2000). Historia del deporte, Zaragoza. Ed. Inde.

SANTIBÁÑEZ, E. et. al. (2000). Sistematización; producción de conocimiento para la acción, Bogotá, Centro de Investigaciones y Desarrollo de la Educación.

SCHÖKEL, L. A. (1994). Apuntes de hermenéutica, Madrid, Ed. Trota.

SCHÖN, D. (1983). Cómo piensan los profesionales en acción: el práctico reflexivo, New York, Basic Books.

TORRES, A. (2002). «Sistematización-Investigación». En: Taller de confrontación: «Seminario-Taller conformación del grupo investigador», proyecto, discursos, saberes $y$ prácticas del deporte contemporáneo en Bogotá y Cundinamarca: una experiencia de sistematización Bogotá, División de Gestión de Proyectos, CIUP, Universidad Pedagógica Nacional, agosto 24 .

ZULUAGA, O. L. (1999). Pedagogía e historia: la historia de la pedagogía. La enseñanza, un objeto de saber, Bogotá, Universidad de Antioquia, Anthropos, Siglo del Hombre Editores. 